\title{
La contaminación heredada: diferentes realidades, distintas soluciones*
}

Sonia Contreras Cascales** http://dx.doi.org/10.21503/lex.v9i8.411

* Ponencia presentada en el Primer Congreso Internacional de Medio Ambiente y Derecho Ambiental, los días 26, 27 y 28 de mayo de 2011, en la Universidad Alas Peruanas Filial Chimbote.

** Abogada y consultora, doctorando en Derecho Ambiental en la Universidad de Alicante en España, profesora de la Escuela de Ciencia Política de la Universidad de Costa Rica, miembro fundador de la Asociación Internacional de Doctores y Doctorando en Derecho Ambiental. 

Tomo es bien conocido, en los últimos 150 años el planeta ha cambiado. La estructura natural de su atmósfera e hidrósfera han padecido más cambios que en todo el tiempo de existencia que lleva.

Se sabe que es fácil y, con frecuencia, inútil caer en la interminable enumeración de problemas y catástrofes ambientales que soporta nuestro planeta. Describir calamidades no es agradable ni para el que las cuenta ni para el que las escucha, pero únicamente la información y la concientización pueden corregir situaciones equivocadas y mitigar sus consecuencias.

Se considera contaminación ambiental a la presencia, en el medio ambiente, de uno o más contaminantes en cantidades superiores a los límites tolerados por el ser humano, combinados de tal manera que, en mayor o en menor medida, causan un desequilibrio ecológico y dañan la salud y el bienestar del ser humano.

La contaminación ambiental se origina generalmente como consecuencia del crecimiento $y$ desarrollo incontrolado de centros de población, turísticos e industriales, con el correlativo incremento de las fuentes de contaminación, el deterioro de los recursos naturales y el impacto de algunos fenómenos del mismo tipo, como las erupciones volcánicas, tolvaneras, fugas tóxicas, entre otros problemas.

Con motivo de la celebración del I Congreso Internacional de Medio Ambiente y Derecho Ambiental y su relación directa con el Caso de Chimbote, es que vamos a centrar la atención en la contaminación producida por actividades económicas como la pesca y la minería, así como su impacto en el medio ambiente.

Como es sabido, la pesca, incluida la acuicultura, constituye una fuente vital de alimentos, empleo, recreación, comercio y bienestar económico para las poblaciones de todo el mundo, tanto para las generaciones presentes como para las futuras y, por lo tanto, debería llevarse a cabo de forma responsable. 
La pesca, como cualquier otra actividad, genera una serie de impactos en el medio ambiente $^{1}$, tales como la explotación excesiva de los recursos, la contaminación del mar, suelo y aire, entre otros. Como la demanda está acercándose a los límites de la producción, muchos recursos pesqueros están sufriendo deterioro. Por un lado, la excesiva pesca está agotando ciertas poblaciones y, por otro, las otras actividades humanas también influyen en la productividad de la pesca en los sistemas de agua dulce, salobre y sala.

Hay que poner atención a la generación de residuos como consecuencia de la actividad pesquera, ya sea a bordo o en los propios puertos, debido al importante grado de contaminación que ocasiona. También, la contaminación procedente de las áreas industriales, urbanas y agrícolas, el uso de la tierra en las cuencas hidrográficas y el manejo de las aguas, son factores que están ejerciendo impactos negativos en la pesca y en el medio ambiente.

Otro fuerte impacto se da con el procesamiento y el transporte del pescado, ya que éste puede requerir una gran infraestructura, incluyendo caminos, instalaciones portuarias y suministro de energía y agua (para hielo, refrigeración, etc).

Por otro lado, la industria minera es uno de los sectores económicos más cuestionados en todo el mundo por ser uno de los principales causantes del deterioro ambiental. La esencia misma de la actividad obliga al ciudadano normal a cuestionar su validez, dado que las huellas son extremadamente visibles: grandes excavaciones, procesos de subsistencia, contaminación de acuíferos y destrucción de suelos. ${ }^{2}$

El impacto potencial que puede ocasionar la minería depende de las características del yacimiento, de la magnitud de las operaciones y de la metodología minera, los equipos y la gestión ambiental. No obstante, debido a la magnitud de determinados proyectos, los impactos sobrepasan el ámbito local y, en muchos casos, llegan a tener incidencia nacional de las políticas macroeconómicas, tributarias, de distribución del ingreso y otras. ${ }^{3}$

Cabe considerar que el concepto de "desarrollo sustentable" es dinámico y que quizás, como pocos, ha evolucionado de manera acelerada, hasta tal punto que se puede observar,

1 Algunos impactos de la actividad pesquera, pueden ser: a) explotación excesiva de las existencias de los peces y degradación a largo plazo de la base de los recursos, b) captura de las especies no objetivos y daños al hábitat, a raíz del uso de ciertos equipos y prácticas de pesca, c) contaminación proveniente de los derrames de petróleo y combustible, y de casco del buque, d) daños causados por los buzos y anclas, e) "Pesca fantasma" y peligros para la navegación, a causa de las redes y trampas abandonadas o perdidas, f) creación o expansión de las áreas portuarias, instalaciones en tierra y obras de infraestructura (caminos, agua, energía eléctrica) para el procesamiento y transporte de los productos de la pesca, g) contaminación proveniente de los efluentes de las plantas industriales de procesamiento de la pesca, etc.

2 CHAPARRO A., Eduardo. Buenas prácticas en la industria minera: El caso del Grupo Peñoles en México. Serie Recursos Naturales e Infraestructura. CEPAL: Santiago de Chile,. 2007, pág. 7.

3 Ibídem., pág. 19. 
con el estudio de algunos casos, como la industria minera responde hoy a los repetidos cuestionamientos que, desde hace algunos años, se han dado a la explotación de minerales en el continente americano.

Pese a la poca difusión que han tenido las respuestas que esta industria ha dado a la preocupación colectiva por un ambiente sano y por prácticas consideradas opuestas a los nuevos esquemas y paradigmas ambientales, sociales y económicos, abundan los ejemplos de cómo esta actividad, insustituible en el panorama económico continental, ha reaccionado, para adoptar normas y códigos de conducta que responden a los temores del público, a las nuevas normas ambientales y a los dictados éticos de conceptos como "cierre de minas", responsabilidad social corporativa" y "comunidades sustentables". ${ }^{4}$

A continuación, se expone la realidad de Chimbote, así como otros casos (con diferentes realidades) donde las actividades mencionadas ocasionaron igualmente impactos ambientales negativos y como fueron las respuestas y/o soluciones adoptadas al respecto.

El Perú no es solo un país minero por excelencia, sino que también tiene una importantísima actividad pesquera. La inmensa biota que existe en su mar hace que sea uno de los primeros países exportadores a nivel mundial. Las industrias pesqueras, en su proceso de producción, producen un alto grado de contaminación en el mar.

En el caso de Chimbote, concretamente, se caracteriza por ser la ciudad pesquera más grande del Perú y es conocida como la "Capital del Trabajo" por el centenar de fábricas que dan empleo a miles de personas y que colocan a Chimbote como primer puerto pesquero en el mundo. Sus principales actividades económicas son la pesca ${ }^{5}$ y la minería ${ }^{6}$ (además de actividades agrícolas y otras industrias), por eso también es conocida como "la Capital de la pesca y el acero"?

Los primeros registros de contaminación en Chimbote comenzaron en los años sesenta, uno de los lados desfavorables que trajo el boom de la pesca. Esto provocó un desmesurado crecimiento de fábricas que hoy en día son la principal fuente de contaminación, puntualmente, los olores que provoca la emanación de humo de las chimeneas de las fábricas pesqueras. Otro de los factores que más influye en la contaminación en Chimbote es la basura que dentro

\footnotetext{
Ibídem., pág. 5 .

Chimbote es conocido por ser un puerto dedicado a la industria pesquera. Las fábricas de harina y aceite de pescado tienen sus plantas industriales en la zona industrial de Chimbote. Las principales especies extraídas son: la anchoveta, el jurel, la caballa y el atún, entre otras.

Chimbote se abastece de las extracciones mineras del interior de la región.

http://www.chimboteperu.com/
} 
de la ciudad constituye uno de los mayores focos infecciosos que existe. ${ }^{8}$ La contaminación ambiental en Chimbote se aprecia fácilmente y su incidencia principal es a nivel marino", atmosférico $^{10}$, del suelo ${ }^{11}$ y urbano. ${ }^{12}$ A continuación, se expone o se plantea algunas de las causas, consecuencias y posibles soluciones a este problema.

Entre las causas principales tenemos la manipulación del pescado, la mala limpieza de las bodegas, la presencia de productos tóxicos y los gases y partículas de la atmósfera. Las posibles consecuencias de estos problemas son la contaminación de la franja costera y las ciudades aledañas, el envenenamiento del agua y del aire, la destrucción del plancton, de la flora y la fauna, la caída del turismo y la proliferación de enfermedades alérgicas. Finalmente, entre las posibles soluciones tenemos la aplicación de leyes que regulen a las empresas pesqueras, mejorar el manejo de la transferencia de pescado y mariscos, tratamiento de aguas servidas, y el lavado de chimeneas.

A continuación, veremos más detalladamente las causas de la contaminación:

1. Manipulación del pescado. Si no se hace un adecuado traspaso de la pesca desde los barcos hasta la industria en tierra, el mar sufre contaminación por materia orgánica y las aguas experimentan una pérdida de oxígeno, proceso que se denomina eutrofización de las aguas. En este caso, los “riles" están constituidos por partículas de grasas, carne y sangre. A consecuencia de la actividad pesquera, caen en sus redes otros peces que no son de utilidad para el consumo.

2. Limpieza de bodegas. Diversos son los modos en que la faena de procesamiento de pescados y mariscos contaminan las aguas del mar. Desde el mismo momento en que se recogen las redes y se almacenan las enormes cantidades de pescados en alta mar, comienza un proceso de contaminación, ya que se acumulan residuos orgánicos en el agua de las bodegas de las naves.

8 http://rschimbote.obolog.com/contaminacion-ambiental-chimbote-108977

9 Contaminación marina: producida por la entrega sin tratamiento previo de los desagües urbanos y por la eliminación de las aguas residuales de las plantas conserveras y de harina de pescado.

10 Contaminación atmosférica: por las emisiones de partículas, gases, vapores y olores que despide la industria pesquera en sus procesos de producción; por la emisión de partículas, gases y vapores de la industria siderúrgica; y por la emisión de gases del antiguo parque automotor de la ciudad.

11 Contaminación del terreno: por la eliminación sin tratamiento, ni clasificación de los desechos urbanos, directamente al terreno y/o a la atmósfera a través de la incineración; por la eliminación de desmontes y materiales orgánicos a la vía pública y terrenos baldíos; por parte de la población, por la eliminación de desechos industriales al terreno, por parte de la industria pesquera y siderúrgica; y la salinización de las pocas tierras agrícolas por malas técnicas de riego.

12 Contaminación urbana: debido al incumplimiento del plan de desarrollo urbano, con una zonificación adecuada; a la escasa arborización y verderización de las calles y parques; a la calidad del agua potable; a la inadecuada manipulación y comercialización de los alimentos; a la construcción convencional de viviendas, sin buscar soluciones acordes con las características físicas-étnicas de la zona. 
3. Presencia de productos tóxicos. En varios estudios realizados para observar los daños que provoca la contaminación de las industrias se obtuvo una mortandad de miles de peces y crustáceos en los mares costeños. En la costa, había aproximadamente 35 especies de peces muertos, tales como lenguado, corvina, jurel y crustáceos. Según las mediciones hechas por las autoridades correspondientes, en los análisis de las primeras muestras de agua contaminada, se detectó la presencia de "formaldehido" en una concentración mayor de la permitida. Este elemento se utiliza en la faena pesquera como desinfectante, ya que elimina gérmenes y patógenos. Produce un daño tóxico directo porque es muy irritante y contribuye a disminuir el oxígeno del agua. En el proceso industrial y para la limpieza de las instalaciones se utiliza soda. Las aguas con soda y restos orgánicos son vertidos directamente al mar sin ningún tratamiento.

4. Gases y partículas a la atmósfera. Para la producción de harina de pescado se utilizan procesos de secado, y los vapores de agua con alta concentración de gases de olor intenso son vertidos al aire, causando contaminación olorosa en las zonas urbanas cercanas, como la contaminación aérea ${ }^{13}$ por parte de trimetilamina y vapores como resultado de la cocción del pescado a altas temperaturas.

Las posibles soluciones a estos problemas son:

Un adecuado manejo de la transferencia de pescados y de los mariscos y de los desechos que se vierten al mar permite que la pesquería sea amigable con el medio ambiente marino.

$\checkmark$ Una solución planteada para disminuir la contaminación por sangre durante las faenas de descarga es una neutralización de las aguas de bombeo, lo que permitiría la concentración de las proteínas solubles y el material orgánico particulado. Estas aguas residuales podrían ser finalmente incorporadas al proceso de producción y no devueltas al medio enriquecidas con materia orgánica.

$\checkmark$ Las sustancias residuales (desechos orgánicos) deben ser recirculadas en el proceso industrial para maximizar el aprovechamiento de la materia orgánica disuelta y evitar la contaminación.

$\checkmark$ Los residuos (líquidos) deben ser tratados de acuerdo a su naturaleza antes de ser vertidos por el colector final.

13 La contaminación aérea por parte de las industrias pesqueras, ocasiona:

$\checkmark$ La contaminación olorosa del ambiente que rodea a estas industrias (ciudades, puertos, etc.).

$\checkmark$ Provoca perjuicios en la salud de la población de las ciudades; tales como: irritación de ojos, infecciones respiratorias, intoxicación, edema pulmonar, entre otros. 
Los gases, como resultado del proceso industrial, deben pasar por un proceso de lavado de chimeneas, cuyo fin es solo eliminar vapor de agua a la atmósfera o debe ser recirculado al proceso industrial con el fin de eliminar el desprendimiento de gases a la atmósfera.

En el caso de España, existe una prolongada plataforma continental que discurre a lo largo de toda la periferia del litoral español. Dicha plataforma, aunque estrecha, ya que el talud se encuentra a escasa distancia de tierra, es muy rica en recursos pesqueros. La explotación de estos recursos marinos tiene una gran tradición en España. ${ }^{14}$

En el Caladero Nacional, faena la mayor parte de la flota pesquera española en cuatro zonas pesqueras diferenciadas: Cantábrico-Noroeste, Golfo de Cádiz, Canarias y Mediterráneo. Se trata de flota de bajura, cuyos buques se encuentran en Censos que les posibilita faenar en zonas determinadas del Caladero y con artes o aparejos específicos, si bien existe un elevado número de unidades pesqueras de carácter artesanal. La elevada demanda de productos pesqueros puso de manifiesto la insuficiencia de la producción pesquera del caladero nacional y propició el desarrollo de pesquerías en aguas exteriores y caladeros lejanos.

El Tratado de la Unión Europea establece que la pesca constituye una de las Políticas Comunes y que, por lo tanto, la Unión tiene competencia exclusiva en la materia. Las Comunidades Autónomas, por su parte, tienen competencia sobre la pesca que se realiza en aguas interiores, el marisqueo y la acuicultura, y en materia de ordenación del sector pesquero y de comercialización de los productos pesqueros, en desarrollo y ejecución del marco unitario. ${ }^{15}$

De entre todas las flotas autonómicas, destaca Galicia con más de 5.000 buques. Es la comunidad autónoma con mayor número de barcos, que suponen casi el $50 \%$ del total de la flota española y el 6\% de la UE. Le sigue Andalucía, con 1.797 pesqueros, que representan el $16 \%$ de la flota española y el 2,09\% de los barcos de la UE. ${ }^{16}$

En el País Vasco ${ }^{17}$, debido principalmente a la presión de los grupos ecologistas en los medios de comunicación de la región en varios puertos, se han llegado a reunir todos los responsables de los diversos sectores relacionados con estos puertos.

14 Ya en la Edad Media y la Edad Moderna, el comercio de sardinas, bacalao y boquerones en salazón, bonito en escabeche etc. estableció redes de conexión entre los puertosgallegos, cantábricos, suratlánticos o del levante, y las ciudades del interior.

15 Este marco viene determinado por la Ley 3/2001, de 26 de marzo, Ley de Pesca Marítima del Estado.

16 GREENPEACE. La Pesca en España: una lección no aprendida. 2010.

17 Gran parte de la historia del País Vasco ha estado profundamente ligada y vinculada al mar. Muchos vascos, han vivido, y siguen viviendo, del mar y para el mar. Las características orográficas del litoral vasco así como una mar viva, recia y llena de contrastes hace que aún sea más difícil la convivencia de estos hombres con las aguas del Golfo de Bizkaia. 
Uno de los problemas ambientales de los puertos vascos es principalmente la contaminación producida por vertidos de combustible al mar, contaminación atmosférica ${ }^{18}$, producción de residuos $^{19}$, contaminación acústica, entre otros.

Ante tal panorama, a continuación se mencionan algunas de las medidas tomadas por las instituciones, concretamente en Ondarroa ${ }^{20}$, después de varios años sin abordar seriamente el problema de la contaminación por parte de las instituciones.

Durante el año 2005, se realizaron varias reuniones en las que tomaron parte tanto el responsable del puerto del Gobierno Vasco, el responsable de la Capitanía de Marina, representantes de las empresas de bajura y altura, embarcaciones de recreo, así como responsables de las empresas suministradoras de gasoil. Debemos criticar la ausencia de los grupos ecologistas, pues fueron ellos los principales abanderados de la lucha contra la contaminación en los puertos, ríos y playas. Asimismo, fue lamentable la ausencia de representantes del Ayuntamiento que, aun no teniendo competencias en la cuestión, sería muy significativa su aportación. ${ }^{21}$

Otra medida será la de no aceptar ningún rebrote en el llenado de los depósitos de gasoil de las embarcaciones. En el caso de suceder este desbordamiento de combustible se deberá dar la alarma a los encargados del puerto. Estas medidas se empezarían a adoptar en los puertos de Ondarroa, Lekeitio y Bermeo.

Para evitar estos vertidos oleosos y de gasoil como cualquier otro tipo de contaminantes y residuos, los representantes de los distintos sectores reunidos solicitan la colaboración de los grupos ecologistas para que lleven a cabo la labor de concienciación entre el personal que realiza trabajos en nuestros puertos, así como entre los responsables de las distintas empresas. Por parte de las empresas armadoras, se deberán cumplir los protocolos para el llenado de gasoil y el vaciado del aceite usado. En el caso de realizarse infracciones, se abrirán expedientes sancionadores a los responsables de estos.

18 Contaminación atmosférica debida al polvo de granalla, pintado de embarcaciones a pistola, vapores de hidrocarburos, las emisiones de gases provenientes de las tuberías de exhaustación de buques, así como a las emisiones de gases provenientes de los tubos de escape del tráfico rodado.

19 Prácticamente la mayoría de los buques de todos los puertos del País Vasco no disponen de un sistema propio de recogida de la basura generada por los arrantzales. Tanto mientras se encuentran faenando, como cuando se encuentran amarrados en puerto.

20 La inversión en la nueva infraestructura del Puerto, así como los amplios servicios ofertados (planta congeladora, remolcador, fábricas de hielo, carros varaderos, grúas, básculas, efectos navales) y las empresas pesqueras exportadoras y conserveras instaladas en el municipio, han conseguido mantener a Ondarroa a la cabeza de la actividad pesquera en el País Vasco, así como adecuarlo a las exigencias demandadas por el mercado.

21 EGUZKI. Puertos Limpios en una Costa Vasca Viva. 
En el año 2006, se sancionó a una embarcación cántabra, Nuestro Padre Tonino, por un vertido de gasoil producido durante la recarga del combustible. Este barco se encontraba amarrado y fue el responsable de la Capitanía de Marina el que realizó la denuncia (25-0406). Este vertido no se hizo voluntariamente, sino que fue debido a una mala alineación de las válvulas, o sea que estaban cerradas, cuando deberían permanecer abiertas. A la empresa armadora de esta embarcación se le sancionó con la cantidad de 6.000 euros.

Aun no siendo nada frecuente este tipo de sanciones, y aun no estando los grupos ecologistas muy de acuerdo con estas políticas sancionadoras, vemos que es un método eficaz para evitar los vertidos contaminantes en los puertos. De hecho, embarcaciones que arrojan todo tipo de residuos al agua de estos puertos, cuando faenan en otros países bien se abstienen de hacerlo, pues saben que serán sancionados por la autoridad portuaria correspondiente.

Además de estas medidas preventivas, es importante destacar las campañas ecologistas de concienciación llevadas a cabo. Como una práctica que tiene lugar cada vez con mayor frecuencia en España, es interesante mencionar el tema de la Ecoetiqueta y la Certificación ${ }^{22}$, así como la responsabilidad social empresarial o corporativa. ${ }^{23}$

En México, el Grupo Peñoles ${ }^{24}$ es una de las empresas más grandes en la industria minera en México, con operaciones integradas en la fundición y afinación de metales no ferrosos y en la elaboración de productos químicos. Asimismo, es el mayor productor mundial de plata finada, bismuto metálico y sulfato de sodio, y líder latinoamericano en producción de oro y plomo, además de ser uno de los más importantes conglomerados industriales y mineros de la república mexicana.

22 Las primeras iniciativas acerca de la calidad de una pesquería determinadasurgieron desde el punto de vista de la comercialización de los productos. Asociaciones de Productores, Cofradías de Pescadores y Autoridades pesqueras, empezaron a emplear en los productos marinos las mismas estrategias comerciales que con los productos agropecuarios: la denominación de origen. Ahora, en determinados Estados miembros de la Unión Europea se intenta establecer un Ecoetiquetado para productos marinos capturados de forma responsable y sostenible. Se trataría de hacer llegar hasta la ciudadanía el mensaje de que los pescados que se adquieren en el mercado están siendo capturados de tal forma que no se pone en peligro el medio ambiente marino y la salud del océano, al tiempo que se garantiza la durabilidad y el equilibrio de la pesquería.

23 En España, la RSC tiene su origen a finales de los años 90 a través de la Asociación de Instituciones de Inversión Colectiva y Fondos de Pensiones (INVERCO) que introduce el concepto de Inversión Social Responsable. Progresivamente, un mayor número de empresas son conscientes de que pueden contribuir al desarrollo sostenible orientando sus operaciones con el fin de favorecer el crecimiento económico y aumentar su competitividad, al tiempo que garantizan la protección del medio ambiente y fomentan la responsabilidad social, incluidos los intereses de los consumidores. Ello, unido a las recientes tendencias de transparencia e información que, en la actualidad se exigen a las empresas (principalmente a aquellas que cotizan en Bolsa), ha dado lugar a que muchas de ellas hayan comenzado a elaborar y publicar informes con las actuaciones responsables en los ámbitos laboral, social y medioambiental que han llevado a cabo durante el año.

24 La Compañía Minera de Peñoles fue fundada en 1887 por un grupo de 18 mineros mexicanos, cuyo propósito era explotar tres minas localizadas en la Sierra de Peñoles, ubicada en el estado de Durango. Hoy esta empresa se conoce como Industrias Peñoles, S.A. de C.V. 
Met-Mex Peñoles, S.A. ${ }^{25}$ es el complejo metalúrgico no ferroso más importante de América Latina y el cuarto a nivel mundial, además de ser el primer productor de plata afinada en todo el mundo. Actualmente, cuenta con tres grandes plantas metalúrgicas en la ciudad de Torreón: la Fundidora de Plomo, la refinería de Plomo-Plata y la Refinería Electrolítica de Zinc. Este complejo cuenta además con dos plantas de ácido sulfúrico, una de óleum, una de sulfato de amonio y una de bióxido de azufre líquido. ${ }^{26}$

Sus principales productos son oro y plata fina, plomo afinado en diferentes grados, plomo antimonial, zinc de alta pureza y aleaciones; cadmio, bismuto, ácido sulfúrico, óleum y litargirio; cobre electrolítico, bióxido de azufre líquido; trióxido de antimonio y sulfato de cobre.

Desde 1994, la empresa se ha sometido a auditorías de carácter voluntario, promovidas por la Procuraduría Federal de Protección Ambiental (Profepa). A su vez, los desarrollos técnicos y legales de protección ambiental han incrementado el rigor de las normas y de los valores límites permitidos. Según la Profepa, hasta febrero de 1999, la empresa había cumplido con 76 de las 113 medidas correctivas, lo que equivale al 67\%.

Posteriormente, se inició un debate público sobre los problemas de contaminación que padecen los habitantes de Torreón y, en particular, los niños de esa ciudad. ${ }^{27}$ Desde el punto de vista ambiental, la otra cara de la moneda de Met-Mex es la contaminación con plomo. ${ }^{28}$

Muchos saben que la solución no era el cierre definitivo de la planta ni su reubicación en otra ciudad -dado que la contaminación está presente en el suelo y en la tierra- sino la posibilidad de que la empresa y la comunidad convivan en armonía, aplicando para ello medidas preventivas más contingentes con el propósito de evitar que haya un niño más contaminado con plomo. ${ }^{29} \mathrm{La}$ crisis desencadenó un plan de acción que, cinco años más tarde, presentaba un panorama muy distinto.

El Plan de Acción que Met-Mex Peñoles ha desarrollado para reducir los niveles de plomo en sangre en los niños, se puede agrupar en 4 áreas principales:

1. Reducción de todo tipo de emisiones. Construcción de grandes bodegas para que toda la

25 La empresa se localiza en le región norte del territorio mexicano conocida como la Comarca Lagunera, en las ciudades de Torreón, Coahuila y Bermejillo, Durango. En esta región, se concentra una de las fuentes de trabajo más importantes: alrededor de dos mil empleados laboran en sus instalaciones, y de ellos dependes económicamente más de diez mil personas.

26 CHAPARRO, Eduardo. Buenas prácticas, pág. 31.

27 Con bastante frecuencia aparecían titulares como: "En Torreón, 34 mil niños envenenados por plomo de Peñoles. Las autoridades y los políticos no los ven, no los reconocen, no son parte de la ciudad; son los olvidados."

28 Los efectos de la exposición prolongada al plomo son: afecta a los sistemas endocrino, cardiovascular, respiratorio, inmunológico y gastrointestinal, además de la piel y los riñones.

29 CHAPARRO A., Eduardo. op cit., pág. 34. 
materia prima y los materiales recirculados se manejaran bajo techo $\left(45.000 \mathrm{~m}^{2}\right)$. Instalación de casa de sacos y precipitadores electroestáticos, plantas de tratamiento de aguas y otros equipos. Se implantó la norma ISO 14000 como sistema de administración ambiental, se incrementó la cobertura de la red de monitoreo ambiental, y se llevaron a cabo diversas actividades para fomentar y profundizar la cultura ambiental en todo el personal.

2. Acciones en el área circundante. Se cambió el suelo superficial, la cubierta de superficies con cemento o pavimento, se aspiraron casas, azoteas, patios, calles y banquetas. Se pavimentaron escuelas primarias, jardines de niños, secundarias y escuelas superiores utilizando concreto hidráulico. Se construyó el parque El Centenario.

3. Acciones en materia de salud. Instalación de 3 centro de atención médica comunitaria (dos fijos y uno móvil) y capacitación a médicos en toxicología ambiental y ocupacional. También se creó en 1999 un fideicomiso (por 60 millones de pesos mexicanos) para asegurar la vigilancia epidemiológica de la población expuesta al plomo.

4. Acciones en materia de comunicación. Diseño e implementación de una campaña de comunicación dirigida a la comunidad.

El caso Peñoles ha sido una experiencia muy rica con grandes enseñanzas para académicos, funcionarios, empresarios y ciudadanos. Ha puesto en evidencia el gran vacío legal y normativo que impide la atención oportuna y eficaz en casos similares. La divulgación de los hallazgos científicos a la población afectada ha sido una herramienta valiosa para promover la conciencia ciudadana.

Entre las soluciones generales contra la contaminación podemos sugerir las siguientes:

1. El Estado ${ }^{30}$ debe preocuparse del problema de la contaminación, dando leyes severas, controlando su cumplimiento y sancionando a los transgresores. El problema ambiental es un problema que afecta al bien común y a la calidad de la vida, y, en consecuencia, no puede quedar al libre albedrío de las personas. El bien común es una responsabilidad del Estado como representante del bienestar de todos los ciudadanos.

2. Una alta responsabilidad incumbe a los gobiernos municipales ${ }^{31}$, responsables directos de la disposición de la basura y las aguas servidas; del control del parque automotor; de las áreas

30 Como principal garante de un ambiente sano, tal y como viene contemplado en la mayoría de las Constituciones Políticas Iberoamericanas.

31 Los gobiernos locales forman parte de la administración descentralizada del Estado y, como tal, los más cercanos a la ciudadanía y sus necesidades. 
verdes; del control de los ruidos molestos; del ornato, y de las emisiones contaminantes en su jurisdicción.

3. Los ciudadanos y las ciudadanas deben tomar más conciencia del problema, exigir respeto por el medio ambiente y no contribuir a su deterioro. El aporte de los ciudadanos, individualmente, puede ser muy grande en algunos aspectos.

4. Se deben usar alternativas menos contaminantes como abonos orgánicos en lugar de los sintéticos; transformar los desechos urbanos orgánicos en abonos; controlar biológicamente las plagas, es decir, combatir los insectos dañinos con sus enemigos naturales, etc.

5. Prohibir la propaganda ciega para los insecticidas, herbicidas y otras sustancias tóxicas, debiéndose alertar obligatoriamente al usuario sobre los efectos contaminantes y letales de las mismas.

6. Educar a la población a través de las escuelas y medios de comunicación (TV, radio, periódicos) en el respeto por el medio ambiente y en la erradicación de pésimas costumbres de contaminación ambiental.

7. Los maestros tienen una muy alta participación en educar a las futuras generaciones hacia la responsabilidad con el medio ambiente y ayudar a la toma de conciencia sobre los daños de la contaminación. 


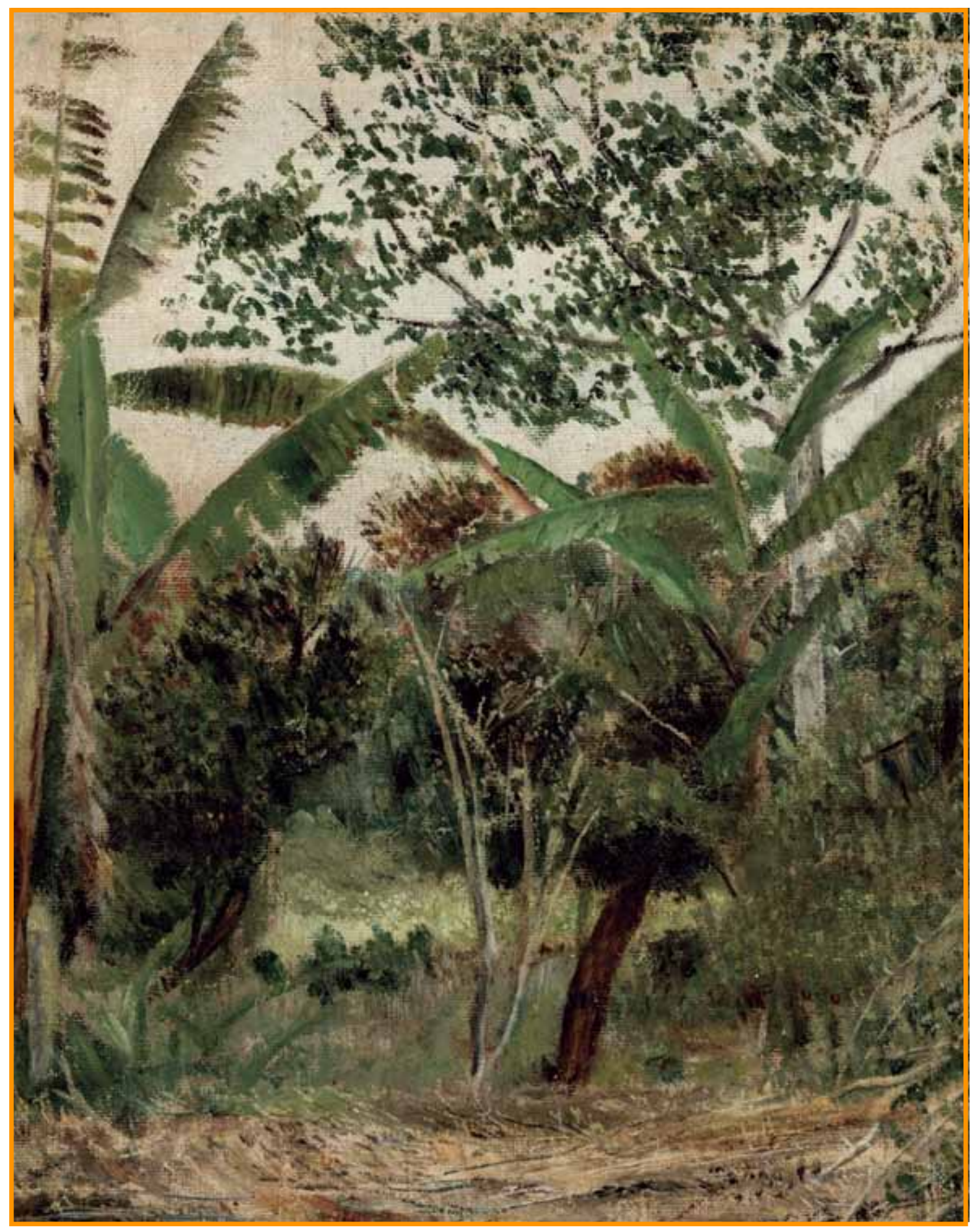

Platanal 